the womb is drawn forwards with the left hand, and a small transverse cut is made at the site of the reflection of the peritoneum on the anterior surface of the uterus (exactly similar to the preliminary peritoneal incision in hysterectomy). With the handle of a knife or with the right index finger, the peritoneum with the bladder is pushed downwards and forwards, until a space of about an inch square is left on the anterior surface of the cervix. The peritoneum on each side of this exposed area is seized with pressure forceps, and is retracted out of the way, while $I$ pass three strong catgut sutures, about one-third of an inch apart, through the denuded surface of the cervix, deep enough to get a firm grip of the cervical muscular coat. The needles having been removed, I draw the ends out respectively on each side through the recti muscles, by the aid of that most handy instrument, Childe's ligature forceps. Thus the peritoneum is excluded, and the exposed cervical connective tissue is brought into direct apposition with the recti. Before tying these three fixation sutures I always close the peritoneal layer of the parietal wound by a continuous catgut suture, the first few loops of which I pass also through the uterine peritoneum just above the denuded area, in order that no saw surface may be left for the omentum or the intestine to adhere to. The three cervix-recti sutures are next tied, and the muscles are approximated through the remainder of the incision by another continuous catgut suture, the rectus sheath is similarly closed, axd the skin is united by a subcuticular silk thread.

In conclusion, I wish to invite attention to a further extract from Cunningham : "The position occupied by the uterus in the pelvis is not always the same, as it varies to some extent with the condition of the neighbouring organs ; the lower cervical part is, however, much more firmly fixed in place than the body and fundus, which possess a considerable amount of mobility.'

I think there cannot be the least doubt that the dominant factor in aterine prolapse is the relaxation of the cervical attachments, and I claim that this simple ten-minute operation is working on anatomical lines in that it leaves the naturally mobile fundus free, and reinforces the immobility of the cervix which is supposed normally to be comparatively fixed, and which is vouchsafed by all anatomists as being the kerstone of support for the pelvic organs.

It is almost superfluous to observe that this method affords direct inspection of the pelvic contents, and an opportunity of dealing with any pathological condition which may coexist. And finally it may be worthy of note that in this operation the two most resistant structures (cervix and recti) in the lower abdomen are utilised.

Buenos Aires.

\section{SOME COMMON FORMS OF DEAFNESS. ${ }^{1}$}

\section{By W. H. KELSON, M.D., B.S. LoND., F.R.C.S. ENG.,} SURGEON AND PATHOLOGIST TO THE LONDON THROAT

$$
\text { HOSPITAL. }
$$

COMMENCING at the external auditory meatus, and working from without inwards, I must first mention cerumen, which, though in many cases it may be quite a simple matter for diagnosis and treatment, has nevertheless sundry traps and pitfalls connected with it. In the first place, although wax generally appears as a yellowish or brownish greasy mass, this is by no means always the case, for it may be hard, horny, and black, and though quite insensitive as a rule it may be surrounded by such an amount of dermatitis, or be 60 closely in contact with the membrana (forming a mould for it in some cases) as to give decided pain on our making ang attempt to move it with the probe. And then, again, one must remember that, particularly where dust and dirt abound, a deceptive appearance is likely to be produced and it may not be wax at all, or only in part, the bulk consisting of dry blood, pus, cholesteatomatous matter, epithelial débris, granulations, polypi, or foreign bodies.

And just one word of warning here. If your patient is markedly deaf-say, for instance, if he cannot hear your watch at all-although you may be certain that cerumen is blocking his meatus, be careful to avoid saying, " $O \mathrm{~h}$, it is

1 Patients illustrating the various kinds of deafness were shown at a demonstration at the Medical Graduates' College and Polyclinic on Feb. 22nd, 1911. only wax, and I can soon put you right," as is often said, for it is quite probable that he has an otitis media or some other disease of the ear in addition to the cerumen, and after removing this latter you will find that you have not cured your patient's deafness, and will then have to climb down somewhat ignominiously.

Having carefully examined the plug with mirror, speculum, and probe, and having come to the conclusion that there is a fair chance of success as regards removal by syringing without previous preparation try to separate the plug from the meatal wall a little with a probe so as to let the stream of water more easily detach it, and then straightening the meatus by drawing the ear somewhat backwards and outwards, direct a stream of water, at a temperature of about $38^{\circ} \mathrm{C}$., into the crack which you have made or found between the plug and the meatal wall. If these efforts are not successful it will be better to desist and dry the ear, and prescribe some drops, remembering that you are dealing with a delicate organ, and that anything like rough or violent syringing is strictly to be avoided.

Now, as to the drops, they may be divided into three varieties: (1) those which contain things which fizz and bubble and so tend to disiniegrate the mass, as peroxide of hydrogen; (2) those having a saponifying effect on the fatty material, such as bicarbonate of soda; (3) those having a softening effect, such as oil and glycerine. Each of these has its supporters, and any will serve. In some cases, especially where the wax is mixed up with hair and epithelial débris, repeated soakings and syringings have to be performed before the meatus can be tboroughly cleared. The after-effects may be somewhat noteworthy. Either the hearing may be hyperacute or even painfully acute, though this gradually planes down to normal; or there may be a comfortable feeling of an obstruction having been removed, with a return of normal hearing. Or (and this is especially so if the case has been an obstinate one, and too much force has been used in syringing) there may be dulness of hearing and tinnitus, which, however, in most cases gradually wear off.

Again, say a patient comes complaining of some dulness of hearing, which has come on recently, especially with a feeling of fulness and throbbing in the ear, with a good deal of pain and tenderness on manipulation of the external ear, and especially on making pressure on the tragus, one may suspect furunculosis, in other words, boils in the meatus. The pain is considerable and quite sufficient often to prevent the patient sleeping, although it is not so deep-seated as in acute otitis media. On examination-which in the first instance must be made by concentrating a strong light on the meatus, but without a speculum, as the introduction of this instrument might prove extremely painful-the walls of the meatus will be found swollen and perhaps in contact, so that no view of the drum can be obtained. The colour may be pale or red, varying with the stage of the disease. and also as to whether it is superficial or deep-seated. On an examination being made very gently with a probe the parts will generally be found to be exquisitely tender, and if suppuration has come to the surface pus will exude. The best treatment will be free incision under nitrous oxide gas, preceded by syringing with carbolic acid, 1 in 20 . If this operation is declined, or the furuncle is freely discharging, and there is no tension whatever, hot applications together with the use of glycerine of carbolic acid will be best. It is well to remember that this disease has a marked relapsing tendency, and that therefore treatment should be kept up and the ear kept under observation considerably after actual symptoms have subsided. Also the general bealth should be attended to and the urine examined. The amount of deafness varies very much in furuncle of the meatus. It may hardly be noticeable, but if the meatus is quite closed by the swelling it is, of course, much more marked.

Before leaving the meatus we must not forget that not infrequently in children, and occasionally in adults, deafness may be due to the presence of a foreign body, when the question, of course, is how to extract this same without damage to the ear. remembering that pain, abscess of the brain, and even death have been caused by rough treatment in endeavouring to extract a foreign body. The first step is carefully to inquire and examine as to the nature and position of the body, and it is well at the outset to instil into the meatus a few drops of a 20 per cent. solution 
of cocaine, or adrenalin. In the vast majority of cases gentle syringing, after a suitable interval, will be the best treatment ; probably 90 per cent. of all foreign bodies can be removed in this way. If this fails, and the patient is restless and the ear tender, especially in the case of young chilaren, a general anæsthetic will be necessary. This having been administered, the syringing should be tried again, and if it fails, one must try one of the numerous hooks, forceps, bent wires, and probes which have been devised with a view to getting hold of or getting behind the body, and so extracting it. Such ideas as those of glueing on a wooden probe to the foreign body, and so extracting it, are more ingenious than practical. In very barl cases, with a sising temperature and signs of deep-seated mischief, the foreign body being hopelessly jammed in the ear, it will be necessary to operate, turning the ear forwards and getting at the deeper points from behind, but these cases are rare.

Passing on now to deafness due to affections of the middle ear, together with which may be put those of the membrana tympani, we first of all note suppuration and its sequelæ. This may begin either in an acute otitis media, such as is commonly seen in scarlet fever and other such diseases, which may either entirely dry up with but little permanent damage to the ear, or destroy the ear as a hearing organ altogether, or become chronic with the formation of granulations, polypi, and carious bone, \&c. ; or it may never pass through the acute stage at all, but be due to some chronic disease, such as tuberculosis.

On examining a suppurating ear we may find, after cleansing, various conditions according to the chronicity of the disease, such as the destruction in whole or part of the membrana tympani and ossicles, caries of the walls, perfora. tions, adhesions, cicatrices, polypi, granulations, and chole. steatomatous matter; and our lines of treatment must be : (1) To see that there is sufficient drainage; (2) to keep the ear clean and remove all dead matter, applying antiseptics, those most generally used being boric acid and alcohol and hydrogen peroxide; and (3) to preserve and possibly improve the hearing. Whether the ear should be syringed or not is rather a moot point, some holding that the treatment tends to aiffuse the disease and in some cases is not necessary. But I certainly hold that the ear cannot always be cleansed without it, but in any case it should be carefully dried afterwards. Suction by means of Siegle's speculum is useful in some cases to extract the discharge.

When symptoms indicate extension of the disease to deeper points, of course more severe measures, such as the radical mastoid operation, have to be considered. One important point must here be noted as regards the hearing, which is not, I think, sufficiently emphasised in books on the subject, and the practitioner should be warned and prepared for itnamely, that as the patient's ear heals and becomes dry, suppuration ceasing, the hearing, much to his annoyance, may get worse; and although later on you may be able to improve this somewhat by inflation, you may have to console him by saying that at any rate a dry ear is infinitely prefer. able to a suppurating one.

Operations for improvement of hearing by division of postsuppurative adhesions unfortunately have given only very poor results; occasionally, however, in such cases where, after suppuration, the hearing is very defective, we may get improvement by means of a so-called artificial drum, and it is certainly worth while making the trial. No elaborate machine or instrument is needed-in fact, these should be avoided. A piece of cotton wool moulded into a small ball, a suitable pair of forceps, an ear speculum and a good light are all that are required. With patience and perseverance you inay perhaps be able to place the ball of wool in such a position, probahly over the head of the stapes, as to markedly improve the patient's hearing, and having found that there is such a spot he will be able himself with care to place the sc-called artificial drum in correct position.

Coming now to non-suppurative affections, we may look upon chronic middle-ear catarrh as one of the commonest causes of deafness. It may be the result of repeated colds in the head or sore-throat, or undue indulgence in tobacco or alcoh sl-in fact, anything likely to set up catarrh, such as adenoid, hypertrophic rhinitis, \&c. The deafness is of ten insidious in onset and varies in severity, bei $g$ worse when the patirnt has a cold and in damp weather. The function of the Eustachian tuke is in abeyance, and the membrana tympani becomes thick and indrawn and often more or less irnmotile and adherent to the deep parts. Inflation per the Eustachian tube may be difficult owing to the swelling of the mucous membranc, and is in the early stages accompanied by squeaking noises and moist sounds. On testing with a tuning-fork, say of middle tone, giving 128 double vibrations per second (C), it will be found that when the fork is placed on the forehearl of the patient he hears it longer in the deafer ear (Weber's test); and if the fork is applied to the mastoid process, and when its vibrations cease to be heard by the patient, if it is placed at his meatus, on the same side but not touching the ear, he will be unable to hear it (Rinne's test, negative) ; or if placed on the vertex he hears it longer than a normal person (Schwabach's test). The patient may or may not hear better in a noise (paracusis Willisii).

As to treatment, in the first place you must inquire into the habits of the patient, with a view to prevention of catarrh, and deal with any post-nasal catarrh and bypertrophic rhinitis there may be present. Alkaline washes to the nose and iodine paint to the post-nasal space are very useful, but above all keep the ear well intlated by Politzer's bag or the catheter. In cases of a particularly obstinate character with Eustachian obstruction, Eustachian bougies are sometimes used, and some surgeons are fond of injecting drugs such as parolein, menthol, \&c., into the midole ear through a Eustachian catheter, or through a cannula passed through it, but the results are apt to be disappointing. The injection of hot air I have several times found to prodnce marked improvement. Electric massage has also been recommended.

Another unfortunately common cause of deafness is the disease known as oto-sclerosis. This is not an affection of the mucous membrane, but a disease of the capsule of the labyrinth consisting of the formation of new bone in some parts and absorption in others, resulting in more or less fixation of the stapes, and often blocking up of the round and oval windows with bony growths. This disease is more common in middle age, but may occur in young or old persons. The cause may be said to be unknown, though the blame has been laid on gout and syphilis. Certainly there seems to be a hereditary tendency in the disease. Tinnitus often precedes the deafness, and is a notable symptom. The deafness is apt to be steadily progressive. The membrana tympani appears practically normal in most cases. Intation can be easily accomplished, but does not improve the hearing. Rinne's test is negative, paracusis well marked. All the above, taken together, give fairly conclusive evidence that we have present a case of oto-sclerosis, the treatment of which distase has so far been very unsatisfactory. Numerous drugs, such as iodide of potassium, phosphorus, and arsenic, have been tried with but little success.

As a less common cause of deafness, there is disease of the internal ear. This may be due to syphilis, malaria, gout, and other diseases tending to produce variations in the intralabyrin!hine pressure. The signs are marked deafness especislly for high notes. There is also bad bone conduction. Rinne's test is positive ; by Weber's test the sound is referred to the better ear, thus giving results opposite to what are found in middle-ear catarrh. The prognosis is bad in these cases, but iodide of potassium and blistering should be tried, and the cause if possible should be found and treated. Cavendish-place, W.

East-End Mothers' Lying-In Hone.-The report of this institution for the year 1910 makes an earnest appeal for increased financial support. The home, which is entirely unsectarian, is maintained for the purpose of treating poor married women during childbirth and also for the training of midwives and nurses for attendance on the poor at their own homes. During the year ending December, 1910 , 558 in-patients were delivered of 561 children borm alive. Three children were stillborn, three in a state of maceration, and there were nine sets of twins. Among the out-patients 1039 women were delivered and 1037 children were born alive. The stillbirths numbered 6 , macerate $\alpha^{3}$ foetus 14, and there were 19 sets of twins. Mr. M. Cursham Corner, the honorary visiting medical officer, states that among the total number of cases attended not a single death has occurred, although a few cases of serious illness have arisen in connexion with the lying-in period. 